\title{
A note on the Radiation Treatment Planning for the Prostate Cancer
}

\author{
Robert Shi* \\ Xinjiang Medical University, Xinjiang, China \\ *Corresponding author: rshi01293@yahoo.com \\ Received January 02, 2014; Revised January 06, 2014; Accepted January 20, 2014
}

\begin{abstract}
Radiation therapy has been one of the major treatment modalities for the prostate cancer over the past few decades. Treatment planning system (TPS) is an integral component in radiation therapy in order to ensure the accurate estimation of radiation dose to the tumor. However, due to the variability in optimization techniques and beam modeling among various TPS, the current literature on radiation treatment planning have shown disagreement on the dosimetric findings, especially for the intensity modulated radiation therapy (IMRT) and volumetric modulated radiation therapy (VMAT). This may create a confusion for the cancer centers who are debating whether to implement IMRT or VMAT as the primary treatment modality for the cancer treatment in their institution. The purpose of this article is to provide the brief review on the IMRT and VMAT for the prostate cancer treatment.
\end{abstract}

Keywords: radiation therapy, treatment planning, prostate cancer

Cite This Article: Robert Shi, "A note on the Radiation Treatment Planning for the Prostate Cancer." American Journal of Cancer Prevention, vol. 2, no. 1 (2014): 1-3. doi: 10.12691/ajcp-2-1-1.

\section{Introduction}

The primary goal of radiation therapy is to enhance the tumor control by delivering maximum dose to it while reducing dose to the normal tissues. Intensity modulated radiation therapy (IMRT) is a treatment technique, which delivers radiation beam either by modulating the beam using continuously moving multi-leaf collimator (MLC) or by dividing the radiation beam into different segments of various MLC shape [1]. Volumetric modulated radiation therapy (VMAT) is relatively a new treatment technique, which delivers radiation beam by variation of gantry speed, dose rate, and MLC leaf positions at the same time [1]. These advanced techniques have enabled to achieve conformal dose distributions to the target volume and reduce the normal tissue dose. One of the major component in radiation therapy is the treatment planning system (TPS), and the plan optimization techniques and dose calculation algorithms play major role in achieving optimal treatment plans in radiation therapy. However, due to the variability in optimization techniques and beam modeling among various TPS, the current literature on radiation treatment planning have shown disagreement on the dosimetric findings. The purpose of this article is to provide the brief review on the IMRT and VMAT for the use of the prostate cancer treatment.

\section{Review of the Literature}

The review of literature on the prostate cancer treatment was done using Google Scholar and PubMed. Since this is a brief review article, the most relevant articles on the IMRT and VMAT are discussed below.

One of the earlier studies by Kjaer-Kristoffersen et al. [2] reported that the results from the VMAT planning were superior than that of the IMRT planning. Additionally, the IMRT planning resulted a higher number of monitor units (MUs) when compared to the VMAT planning [2]. In agreement with Kjaer-Kristoffersen et al. [2], a study by Zhang and colleagues [3] also demonstrated the superiority of the VMAT, especially for the rectal wall. Similar result was reported by Rao et al. [4] and Sze et al. [5] showing better dosimetric quality using the VMAT than using the IMRT. Recently, Herman et al. [6] published their dosimetric results on prostate cancer reporting very minimal difference between the VMAT and IMRT plans. Tsai et al. [7] also showed no clinically significant different between the VMAT and IMRT planning. In contrast to the findings of above mentioned studies [2-7], several research groups have reported that the IMRT planning is better than the VMAT planning for the prostate cancer. For example, Wolff and colleagues [8] demonstrated lower rectal doses in the IMRT plans than in the VMAT plans. Furthermore, Yoo et al. [9] also showed that the VMAT planning produced lower doses for the bladder, small bowel, and rectum.

\section{Discussion}

It is evident that the previous treatment planning studies [2-9] comparing the VMAT and IMRT for the prostate cancer have produced inconsistent results. Such inconsistency may be attributed to the difference in the TPS used by various research groups. The final dosimetric 
results in the cancer treatment plans are typically obtained after the plan optimization followed by the dose calculations. Hence, it is possible to get the different dosimetric results if the plan optimization technique of one research group is different than that of another. During the inverse plan optimization, which are used for the IMRT and VMAT, the treatment planning personnel can assign the weightings for each structure depending on the dose constraints. The plan optimization can be repeated until the optimal plan is achieved; however, treatment planning time could be an important issue for the busy clinics. Hence, the dosimetric results can also be dependent on the experience of the treatment planning personnel.

While the dosimetric quality of the IMRT and VMAT is still a research topic, a number of studies investigated the VMAT planning techniques. The literature shows that the dosimetric results from the VMAT are dependent on the number of arcs used for the planning. For example, Chow and Jiang [10] found that double-arc technique produced lower dose to the rectum and bladder when compared to the single-arc technique. Rana and Cheng [11] also reported similar result showing better dosimetric quality of the double-arc technique. The double-arc technique, however, has a longer treatment time compared to the single-arc technique. [5,10] It has also been suggested the use of partial-single-arc technique in an effort to reduce the rectal dose from the standard full single-arc technique [12].

Dose calculation algorithms employed in the TPS can affect the dosimetric results of the cancer treatment plans [13]. This is mainly because of the difference in beam modeling within the dose calculation algorithm while accounting tissue heterogeneity corrections and scatter dose contribution [13,14,15]. Another factor that can impact the treatment planning results is the photon beam energy $[16,17,18]$. Recently, Pokharel [16] has shown that selection of photon energy (6 MV versus $15 \mathrm{MV}$ ) can affect the normal tissue dose for the VMAT planning of prostate cancer treatment plan. Furthermore, the mixedenergy plans could provide better dosimetric results than the plans calculated using single energy (6 MV or $15 \mathrm{MV}$ ) [16]. However, one should be aware of the neutron dose associated with a higher photon energy [19]. Currently, most of the treatment planning optimizations are carried out using the dose-volume objectives. The use of radiobiological models for the plan optimizations will provide valuable information, which can be used to predict the treatment outcome of the patients $[13,20]$. Since radiation therapy is often combined with the chemotherapy [21,22], the results from such combination will be useful for the clinical evaluation.

\section{Conclusion}

Due to several factors involved in the planning process of prostate cancer treatment plans, it is almost impossible to determine which modality is ideal one for the prostate cancer treatment. Further studies must be conducted to report the clinical results such as normal tissue toxicities and tumor control. Clinical results must be correlated to the dosimetric findings obtained from different treatment planning techniques.

\section{References}

[1] Otto K. Volumetric modulated arc therapy: IMRT in a single gantry arc. Med Phys 2008; 35: 310-17.

[2] Kjaer-Kristoffersen F, Ohlhues L, Medin J, Korreman S. RapidArc volumetric modulated therapy planning for prostate cancer patients. Acta Oncol. 2009; 48 (2): 227-32.

[3] Zhang P, Happersett L, Hunt M, Jackson A, Zelefsky M, Mageras G. Volumetric modulated arc therapy: planning and evaluation for prostate cancer cases. Int J Radiat Oncol Biol Phys. 2010 Apr; 76 (5): 1456-62.

[4] Rao M, Yang W, Chen F, Sheng K, Ye J, Mehta V, Shepard D, Cao D. Comparison of Elekta VMAT with helical tomotherapy and fixed field IMRT: plan quality, delivery efficiency and accuracy. Med Phys. 2010 Mar; 37 (3): 1350-9.

[5] Sze HC, Lee MC, Hung WM, Yau TK, Lee AW. RapidArc radiotherapy planning for prostate cancer: Single-arc and doublearc techniques vs. intensity-modulated radiotherapy. Med Dosim 2012; 37: 87-91.

[6] Herman TF, Schnell E, Young J, Hildebrand K, Algan Ö, Syzek E, Herman T, Ahmad S. Dosimetric comparison between IMRT delivery modes: Step-and-shoot, sliding window, and volumetric modulated arc therapy-for whole pelvis radiation therapy of intermediate-to-high risk prostate adenocarcinoma. J Med Phys 2013; 38: 165-72.

[7] Tsai CL, Wu JK, Chao HL, Tsai YC, Cheng JC. Treatment and dosimetric advantages between VMAT, IMRT, and helical tomotherapy in prostate cancer. Med Dosim. 2011 Autumn; 36 (3): 264-71.

[8] Wolff D, Stieler F, Welzel G, Lorenz F, Abo-Madyan Y, Mai S, Herskind C, Polednik M, Steil V, Wenz F, Lohr F. Volumetric modulated arc therapy (VMAT) vs. serial tomotherapy, step-andshoot IMRT and 3D-conformal RT for treatment of prostate cancer. Radiother Oncol. 2009; 93 (2): 226-33.

[9] Yoo S, Wu QJ, Lee WR, Yin FF. Radiotherapy treatment plans with RapidArc for prostate cancer involving seminal vesicles and lymph nodes. Int J Radiat Oncol Biol Phys. 2010 Mar 1; 76 (3): 935-42.

[10] Chow JC, Jiang R. Prostate volumetric-modulated arc therapy: dosimetry and radiobiological model variation between the singlearc and double-arc technique. J Appl Clin Med Phys. 2013 May 6; 14 (3): 4053.

[11] Rana SB, Cheng C. Investigating VMAT planning technique to reduce rectal and bladder dose in prostate cancer treatment plans. Clin Cancer Investig J 2013; 2: 212-7.

[12] Rana S, Cheng C. Feasibility of the partial-single arc technique in RapidArc planning for prostate cancer treatment. Chin J Cancer. 2013; 32 (10): 546-52.

[13] Lu L. Dose calculation algorithms in external beam photon radiation therapy. Int J Cancer Ther Oncol 2013; 1 (2): 01025.

[14] Oyewale S. Dose prediction accuracy of collapsed cone convolution superposition algorithm in a multi-layer inhomogenous phantom. Int J Cancer Ther Oncol 2013; 1 (1): 01016.

[15] Rana S, Rogers K. Dosimetric evaluation of Acuros XB dose calculation algorithm with measurements in predicting doses beyond different air gap thickness for smaller and larger field sizes. J Med Phys. 2013; 38 (1): 9-14.

[16] Pokharel S. Dosimetric impact of mixed-energy volumetric modulated arc therapy plans for high-risk prostate cancer. Int J Cancer Ther Oncol 2013; 1 (1): 01011.

[17] Park JM, Choi CH, Ha SW, Ye SJ. The dosimetric effect of mixed-energy IMRT plans for prostate cancer. J Appl Clin Med Phys 2011; 12: 3563.

[18] Hawke S, Torrance A, Tremethick L. Evaluation of planned dosimetry when beam energies are substituted for a fraction of the treatment course. Int J Cancer Ther Oncol 2013; 1 (2): 01014.

[19] Kry SF, Salehpour M, Followill DS, et. al. The calculated risk of fatal secondary malignancies from intensity modulated radiation therapy. Int J Radiat Oncol Biol Phys 2005; 62: 1195-203.

[20] Mavroidis P. Clinical implementation of radiobiological measures in treatment planning. Why has it taken so long? Int J Cancer Ther Oncol 2013; 1 (1): 01019.

[21] Sanfilippo N, Hardee ME, Wallach J. Review of chemoradiotherapy for high-risk prostate cancer. Rev Recent Clin Trials. 2011 Jan; 6 (1): 64-8. 
[22] Saha A, Chattopadhyay S. Assessment of pulmonary toxicities in breast cancer patients undergoing treatment with anthracycline and taxane based chemotherapy and radiotherapy-a prospective study. Int J Cancer Ther Oncol 2013; 1 (2): 01021. 